\title{
A Short Pediatric Crohn's Disease Activity Index for Quality Improvement and Observational Research
}

\author{
Michael D. Kappelman ${ }^{1}$, Wallace V. Crandall ${ }^{2}$, Richard B. Colletti ${ }^{3}$, Anthony Goudie ${ }^{4}$, lan H. \\ Leibowitz $^{5}$, Lynn Duffy ${ }^{5}$, David E. Milov ${ }^{6}$, Sandra C. Kim ${ }^{1}$, Bess T. Schoen ${ }^{7}$, Ashish S. \\ Patel $^{8}$, John Grunow ${ }^{9}$, Evette Larry ${ }^{4}$, Gerry Fairbrother ${ }^{4}$, and Peter Margolis ${ }^{4}$ on behalf of \\ the ImproveCareNow Collaborative for Pediatric IBD \\ ${ }^{1}$ Department of Pediatrics, University of North Carolina at Chapel Hill, Chapel Hill, NC \\ 2 Division of Gastroenterology, Nationwide Children's Hospital, Columbus, Ohio \\ ${ }^{3}$ Department of Pediatrics, University of Vermont, Burlington, VT \\ ${ }^{4}$ Child Policy Research Center, Cincinnati Children's Hospital Medical Center, Cincinnati, OH \\ ${ }^{5}$ Inova Pediatric Digestive Disease Center, Fairfax, VA \\ ${ }^{6}$ Nemours Children's Clinic, Orlando, FL \\ 7 Department of Pediatrics, Emory University, Atlanta, GA \\ ${ }^{8}$ Division of Gastroenterology, Children's Medical Center of Dallas, Dallas, TX \\ ${ }^{9}$ Department of Pediatrics, University of Oklahoma, Oklahoma City, OK
}

\section{Abstract}

Background-Practical and objective instruments to assess pediatric Crohn's disease (CD) activity are required for observational research and quality improvement.

Objectives-1) To determine the feasibility of completing the Pediatric Crohn's Disease Activity Index (PCDAI) and the Abbreviated PCDAI (APCDAI), and 2) To create a short PCDAI by retaining and re-weighting the most practical and informative components.

Methods-Physicians in the ImproveCareNow Collaborative for pediatric inflammatory bowel disease (IBD) were asked to record components of the PCDAI and assign a Physician Global Assessment (PGA) of disease severity at each patient encounter. We assessed the feasibility of the PCDAI, the APCDAI, and the individual index components by determining the proportion of visits in which data were recorded. We created a short index by retaining and reweighting components of the PCDAI completed in $\geq 80 \%$ of visits. The feasibility of the short PCDAI and its ability to discriminate between PGA categories were evaluated using descriptive statistics.

\footnotetext{
Results-This study population included 1355 subjects with CD (6373 visits). The PCDAI and APCDAI were complete in $16.7 \%$ and $44.1 \%$ of visits respectively. A short PCDAI, including general well-being, abdominal pain, stools, weight, abdominal exam, and extra-intestinal manifestations could be completed in $66.5 \%$ of visits. The correlation between the Short PCDAI and PGA was similar to that of the PCDAI ( $r=0.60, p<0.001$ vs $0.61, p<0.001)$.
}

Information for correspondence: Michael Kappelman, MD, MPH, University of North Carolina Chapel Hill, Department of Pediatrics, Division of Pediatric Gastroenterology, 130 Mason Farm Road, campus box 7229, Chapel Hill, NC 27599, Phone: (919) 966-1343, Fax: (919) 966-8641, michael_kappelman@med.unc.edu.

Conflict of Interest: No authors have a conflict of interest to disclose 
Conclusion-The Short PCDAI is a practical and valid tool to measure pediatric CD activity disease activity. Its use should facilitate quality improvement and observational research.

\section{Background}

Crohn's disease (CD) is a chronic, relapsing disorder of the gastrointestinal tract which affects nearly 600,000 Americans[1], including 50,000 children [2]. Nearly $25 \%$ of patients experience onset of their disease during the childhood or adolescent years. Because childhood-onset disease is associated with a high degree of patient morbidity including missed work [3] and school[4], decreased quality of life[5,6], and psychosocial disturbance[7,8], both clinical research and quality improvement approaches are necessary to improve outcomes for affected patients.

A significant impediment to research in pediatric Crohn's disease has been the difficulty in selecting an appropriate outcome measure $[9,10]$. Disease activity indices, such as the Pediatric Crohn's Disease Activity Index (PCDAI) have been developed and validated as endpoints for clinical trials[11]. However, due to problems inherent in both the funding and recruitment for clinical trials (i.e. interventional studies), much of the research in pediatric Crohn's disease has relied on observational study designs such as patient registries and retrospective chart reviews. Because the PCDAI requires determination of height velocity, analysis of laboratory tests, and components of the physical exam (perirectal examination) that are not always completed during patient encounters, the feasibility of this index in observational studies has been called into question[12]. Consequently, a disease activity index that can be easily completed in the context of routine clinical care would greatly facilitate clinical research in pediatric Crohn's disease. In addition, a more practical index would also be helpful in measuring outcomes for quality improvement purposes, in establishing performance benchmarks, and in assisting individual clinicians at the point of care in making decisions about whether or not to step-up or adjust medical therapy. Shortened activity indices have been previously developed for adult Crohn's disease[13] and ulcerative colitis[14] assessments, with good feasibility and overall accuracy. Although an abbreviated PCDAI (APCDAI) has been previously proposed[15] and tested in a single center study[16], this instrument has not been evalulated in a large, multi-center setting. Furthermore, use of the APCDAI in observational research may be limited by the requirement of a perirectal examination which is not always performed during routine clinic visits.

The aims of this study were 1) To determine the feasibility of completing the PCDAI, APCDAI, and their individual components in the context of routine clinical care, and 2) To create a Short PCDAI by retaining and re-weighting the most practical and informative components. To accomplish this, we performed an analysis of clinical and laboratory data prospectively collected by centers participating in the ImproveCareNow Collaborative, a quality improvement project for Crohn's disease and ulcerative colitis (formerly known as the PIBDNet Trailblazer Improvement Collaborative).

\section{Methods}

\section{Study population}

The ImproveCareNow Collaborative for pediatric inflammatory bowel disease (IBD) was formed in April 2007 by 9 participating pediatric gastroenterology programs. Subsequently, an additional 6 centers joined the network. In this quality improvement program, children with previously and newly diagnosed IBD were enrolled during clinic visits, and followed prospectively at each encounter. At the onset of the collaborative, a case report form was designed to capture clinical and demographic information about each patient, including all 
of the components of the PCDAI as well as a Physician Global Assessment (PGA) of disease severity. Physicians at participating sites were asked to complete each of these items at each clinic visit.

\section{Determination of PCDAl scores}

For each patient visit, we scored each component of the PCDAI if sufficient data were available. If the required data were not available, we considered the component as incomplete and a score was not assigned. PCDAI component scores were assigned using the methods described in the published user's guide[10]. We calculated height velocity Z scores based on Centers for Disease Control (CDC) height velocity growth charts, only if a prior height and date were previously recorded 6-12 months prior to that visit. We based all Z scores on chronological age, as bone age and Tanner staging were not available for all patient visits. For female patients age 15 years or greater, or male patients age 17 years or greater, the height velocity PCDAI component was assigned as score of " 0 " because even a height velocity of $0 \mathrm{~cm} /$ year was greater than $1 \mathrm{SD}$ below the mean. For the weight component, we used a historic weight recorded 4-6 months prior to the visit in order to assign the PCDAI weight score. We did not take into account weight loss which may have occurred due to corticosteroid withdrawal.

\section{Data analysis}

We used standard descriptive statistics to summarize the demographic characteristics of included patients. We then assessed the feasibility of measuring the PCDAI, the APCDAI, and the individual components of the PCDAI in the context of routine clinical care by determining the proportion of visits for which each index (or individual component) could be assigned a score. Next, we evaluated the relationship between the PCDAI and Physician Global Assessment (PGA) in our study population. For visits in which each of the components of the PCDAI and the PGA were completed, we used box and whisker plots and descriptive statistics to analyze the distribution of PCDAI scores across the following categories of PGA: inactive, mild, moderate, and severe.

In order to create a short index with improved feasibility, we examined the distribution of responses for each component of the PCDAI and retained only those components that were completed in $\geq 80 \%$ of visits. This led to removal of the items that were more often incomplete including perirectal exam, height velocity, and laboratory values. Thus, our final short index, included the following 6 components of the original PCDAI: abdominal pain, patient functioning/general well-being, stools, weight, abdominal exam, and extra-intestinal manifestations.

Next, to determine whether re- weighting of any of the individual components would improve the overall accuracy of the short index, we randomly split the visits into a training set and a validation set. In the training set, we created linear regression models using each of the component scores as independent variables and the log-transformed full PCDAI score as the dependent variable. Log transformation of the full PCDAI score was necessary as these scores were not normally distributed. A value of 0.5 was added to the full PCDAI score values prior to $\log$ transformation to account for values of 0 . The components of the short index that were most strongly associated with the log-transformed total PCDAI score were identified from the size of the standardized regression parameters in the fitted multivariable model. Standardized regression parameters, rather than unstandardized $\beta$ coefficients, were utilized to account for differences in standard errors between each of the components. These components were then weighted according to the magnitude of the standardized regression coefficients. 
We then validated this component weighting strategy in the validation set using a two step process. First, we confirmed that the three items identified for weighting in the training set had similar standardized regression parameters in the validation set. Next, we compared the weighting schema selected in the training set with alternative weighting schema using Pearson's correlation to measure the association between each schema and the logtransformed PCDAI in the validation set. We decided, a. priori, that if the correlation of the weighting schema identified in the training set was equal to or greater than that of the alternative weighting schema in the validation set, we would retained the original weighting strategy assigned during the training phase. To confirm that the selected weighting schema was not overly influenced by repeated measures within individual subjects, we repeated the training and validation procedures using only the first visit for each eligible subject and obtained similar results.

Finally, we evaluated the feasibility and performance of the Short PCDAI in the full set of visits. We assessed the overall feasibility of the Short PCDAI by determining the proportion of total visits for which the Short PCDAI could be assigned a score. We then measured the correlation between the Short PCDAI and a number of other indicators of disease severity including PCDAI, abbreviated PCDAI, PGA, hemoglobin, ESR, and albumin. Pearson's correlation was used to assess correlation with log transformed continuous measures (PCDAI and abbreviated PCDAI), and Spearman correlation was used for ordinal variables (PGA, lab parameters) We further characterized the distribution of Short PCDAI scores across different PGA categories using means (s.d.), medians (IQ range), and box and whisker plots. In addition, we used receiver operating characteristic (ROC) curves to test the discriminant ability of the Short PCDAI and other indices to distinguish between inactive and mild disease and between mild and moderate-severe disease. Finally, we calculated the sensitivity and specificity of the Short PCDAI for discriminating between inactive and mild disease and between mild and moderate-severe disease at different cut-points.

All statistical analyses were performed using SAS version 9.2 (SAS Institute: Cary, NC). The study protocol was approved by all sites participating in the collaborative, and informed consent was obtained if required by institutional IRB.

\section{Results}

\section{Patient Demographics}

This study included a total of 1355 subjects with Crohn's disease, comprising a total of 6373 visits. The mean age was 14.2 years (SD. 3.3). Fifty-six percent were male, and the racial/ ethnic distribution was as follows: $74 \%$ white, $10 \%$ black, $2 \%$ Hispanic, and $14 \%$ not identified or other. The mean number of visits per patient was 4.5 (s.d. 3.6).

\section{Feasibility of PCDAI and APCDAI}

The PCDAI scores for each component of the index, as well as the proportion of visits for which each component could be assigned a score, is displayed in Table 1. All 11 components of the PCDAI were complete in only 1067 of the 6373 visits (16.7\%). The abbreviated PCDAI was completed in 2811 out of 6373 visits (44.1\%). PCDAI components completed in less than $80 \%$ of visits included albumin, hemoglobin, sedimentation rate, height velocity, and perirectal examination.

\section{Relationship of PCDAI Score and Physician Global Assessment}

The distribution of PCDAI scores across the following categories of PGA: inactive, mild, moderate, and severe are displayed as box and whisker plots in Figure 1a. The mean (s.d.) PCDAI scores (0-100 scale) for patients with inactive, mild, moderate, and severe disease 
were 7.0 (7.1), 18.3 (10.8), 26.2 (11.4), and 40.5 (14.2), respectively. As shown in the figure and consistent with prior literature, there is overlap of PCDAI scores across PGA categories, but the PCDAI is a reasonable instrument to discriminate between PGA categories.

\section{Development of a Short PCDAI}

Retaining all elements of the abbreviated PCDAI with a feasibility $\geq 80 \%$ resulted in a short index containing the following components: abdominal pain, patient functioning/general well-being, stools, weight, abdominal exam, and extra-intestinal manifestations. In the training set, three items (patient functioning/general well-being, abdominal pain, and weight) were most strongly associated with the log-transformed total PCDAI score (standardized regression parameters approximately twice that of the other components), and considered as candidate items to receive a higher weighting. Based on this, a value of 20 points was selected for these three items (twice the weight of the other components) (Table 2 ). To validate this weighting strategy, we confirmed that 1 ) these components had the highest standardized regression parameters in the validation set, and 2) that the Pearson correlation between the re-weighted Short index and the log-transformed full PCDAI was equal to or greater than several alternative weighting strategies (data not shown). After repeating the training and validation procedures using only the first visit for each subject, we confirmed that the optimal component weighting did not change, suggesting that the weighting schema selected was not overly influenced by repeated measures within individual subjects.

\section{Evaluation of the Short PCDAl}

Overall, the Short PCDAI could be completed in 4241 out of 6373 visits, resulting in feasibility $66.5 \%$ (compared with $16.7 \%$ for the full index and $44.1 \%$ in the abbreviated index). The correlation between the Short PCDAI and disease severity assessed by PGA was similar to that of the full PCDAI $(r=0.60, \mathrm{p}<0.001$ vs $0.61, \mathrm{p}<0.001)$. The correlation between the Short PCDAI and the log transformed PCDAI and Abbreviated PCDAI were 0.66 and 0.84 respectively. Correlations with laboratory indicators of disease activity are shown in Table 3. Box and whisker plots demonstrating the relationship between the Short PCDAI and PGA are shown in figure 1b. The mean (s.d.) Short PCDAI scores for patients with inactive, mild, and moderate to severe disease are 6.1 (8.7), 19.5 (13.3), 31.6 (17.1), and 47.3 (16.0), respectively. Additionally, the area under the curve values generated from receiver operating characteristic (ROC) curves demonstrating the discriminant ability of the Short PCDAI, PCDAI, and ABPCDAI in distinguishing between inactive and mild disease and between mild and moderate-severe disease are displayed in Table 4. Discriminatory capacity for the Short PCDAI is approximately the same as for the two expanded indices. Finally, Table 5 shows the sensitivity and specificity of the Short PCDAI in discriminating between inactive and mild disease and between mild and moderate-severe disease at different cut-points. For discrimination between inactive and mild, we recommend a cutpoint of less than 15. For discrimination between moderate-severe and mild, we recommend a cut-point of $\geq 30$.

\section{Discussion}

In this large, multi-center study, we found that the completion of the Pediatric Crohn's Disease Activity Index and the Abbreviated PCDAI in the context of routine clinical care was quite limited. The components of height velocity, laboratory data, and perirectal examination were the major barriers to completion of these indices. We therefore created a Short PCDAI by eliminating these components, and re-weighting the remaining components to maximize the correlation of the Short PCDAI and the PCDAI. As compared to the full index, this short index is more feasible to complete in the context of routine clinical care and 
has similar correlation to physician global assessment. The ability to discriminate between inactive and mild disease and between mild and moderate-severe disease is also similar to the full index.

Based on these properties, we believe the Short PCDAI is a promising tool for quality improvement and observational research. It should allow providers to track their patient outcomes over time in a more objective fashion, study how changes in their practices impact patient outcomes, and even allow comparisons to be made to national performance standards (i.e. benchmarking). In addition, this instrument should facilitate retrospective chart reviews and prospective registry studies. Given the myriad of financial and ethical issues, along with the practical issue of difficult recruitment, registries and other observational studies have been a mainstay of clinical research in pediatric IBD for decades. Until now, a significant limitation of such observational studies is the large amount of missing data resulting in possible informational bias when PCDAI was used as an outcome measure, or the inherent subjectivity incurred when Physician Global Assessment was the primary outcome. Hence, the Short PCDAI may optimize the balance between feasibly and objectivity that is necessary for observational research.

Our results regarding the limited ability to complete the PCDAI in the context of routine clinical care are consistent with prior published studies. Turner et al recently published a study indicating that the PCDAI could be completed in less than 50\% of visits[12]. The feasibility of the PCDAI reported in that study was higher than we report, perhaps owing to differences in study design and setting. Turner's study included patients in the Pediatric IBD Collaborative Research Group, a select group of closely followed newly diagnosed children with IBD. In contrast, rather than a "research study" where careful procedures were used to optimize the completeness of data collected on a limited number of selected patients at selected center, this was a study of actual clinical practice. Participating centers sought to enroll 100\% of their patients with Crohn's disease, and the data in the ImproveCareNow database reflect that captured during routine clinic visits. Therefore, it is not surprising that the completeness of the different clinical indices reported in this study are lower than that reported in other studies. In fact, this underscores the difference between the feasibility of completing an index for a specific research study and the feasibility of completing an index in actual clinical practice.

In our study, we also observed variation in the completeness of PCDAI components across centers. This study included 15 centers, with the largest center contributing approximately $25 \%$ of included patients. Therefore, it is possible that this and other large centers might have heavily influenced our results. However, the diversity of sites in the ImproveCareNow collaborative reflect the settings in which pediatric IBD care is delivered in the real world (large and small centers, private and academic practices, etc.)

Our short index differs from the APCDAI in that it does not require the perirectal examination. Prior work has suggested that this component helps discriminate between remission and recurrence with a sensitivity of $32 \%$ and a specificity of $98 \%$ [15]. We chose to eliminate this item from our short index because this component of the physical examination is neither indicated nor routinely completed at all outpatient visits. Furthermore, inclusion of this element did not substantially improve the correlation with the full index. Thus, we believe the overall benefit of increased feasibility achieved by eliminating the perirectal exam component is worth this minor trade-off.

It was not possible to select an a. priori threshold for which items to retain in the Short PCDAI. Rather, this threshold was decided after examining the distributions of responses for each component item. If we included completed at less than $80 \%$ of visits, the ability to 
complete the Short PCDAI during routine clinical care would fall to less than two-thirds, greatly limiting the utility of the index. Had we required higher completion rates, this would have eliminated items which are clinically very important and components of most other indices for pediatric and adult IBD (stools, general well-being). Hence, this threshold was selected in order to balance completeness and clinical relevance.

As with the parent PCDAI, the ability of the Short PCDAI to discriminate between inactive and mild disease and between mild and moderate-severe disease is somewhat limited (Figure 1A and B). This is in part due to the fact that Physician Global Assessment is not a true "gold standard". Although used as a gold standard in this and other studies evaluating clinical indices in IBD, the subjectivity of this reference limits the ability to actually measure sensitivity and specificity. In a recent evaluation of the PCDAI, the sensitivity and specificity in distinguishing inactive from mild disease was 0.81 and 0.68 respectively, and in distinguishing between mild and moderate-severe was 0.71 and 0.83 respectively[17]. In the present study, the discriminatory capability of the Short PCDAI was similar, though slightly decreased. This is perhaps due to the fact that PGA was obtained from a large number of practicing clinicians across 15 centers. Although this is a major strength of this study, to the extent that assignment of PGA varies across providers, the sensitivity and specificity of both the full and short index will be diminished. Therefore, the values reported here likely represent a worst-case scenario. In summary, existing pediatric Crohn's disease activity indices including the PCDAI and APCDAI are of limited value during routine clinical care, as many of the required elements are not always collected and recorded during patient encounters. By retaining and re-weighting the most frequently collected items, we have developed a Short PCDAI that is easier to complete at the point of care, has excellent correlation to the full index, and appears to have a similar ability as the PCDAI to discriminate between different levels of disease activity as measured by Physician Global Assessment. We expect this standardized classification tool will facilitate observational research, quality improvement, and clinical care.

\section{Acknowledgments}

Study Support: Dr. Kappelman was supported in part by the National Center for Research Resources (NCRR) Grant KL2 RR025746 and the National Institute for Diabetes and Digestive and Kidney Diseases Grant P30 DK034987. Support for this analysis was also provided by the the Agency for Healthcare Research and Quality grant HS 016957.

\section{References}

1. Loftus EV Jr, Schoenfeld P, Sandborn WJ. The epidemiology and natural history of Crohn's disease in population-based patient cohorts from North America: a systematic review. Aliment Pharmacol Ther. 2002; 16(1):51-60. [PubMed: 11856078]

2. Kappelman MD, et al. The prevalence and geographic distribution of Crohn's disease and ulcerative colitis in the United States. Clin Gastroenterol Hepatol. 2007; 5(12):1424-9. [PubMed: 17904915]

3. Longobardi T, Jacobs P, Bernstein CN. Work losses related to inflammatory bowel disease in the United States: results from the National Health Interview Survey. Am J Gastroenterol. 2003; 98(5): 1064-72. [PubMed: 12809829]

4. Ferguson A, Sedgwick DM, Drummond J. Morbidity of juvenile onset inflammatory bowel disease: effects on education and employment in early adult life. Gut. 1994; 35(5):665-8. [PubMed: 8200562]

5. Akobeng AK, et al. Quality of life in children with Crohn's disease: a pilot study. J Pediatr Gastroenterol Nutr. 1999; 28(4):S37-9. [PubMed: 10204523]

6. Cohen RD. The quality of life in patients with Crohn's disease. Aliment Pharmacol Ther. 2002; 16(9):1603-9. [PubMed: 12197839] 
7. Szigethy E, et al. Depressive symptoms and inflammatory bowel disease in children and adolescents: a cross-sectional study. J Pediatr Gastroenterol Nutr. 2004; 39(4):395-403. [PubMed: 15448431]

8. Mackner LM, Crandall WV. Long-term psychosocial outcomes reported by children and adolescents with inflammatory bowel disease. Am J Gastroenterol. 2005; 100(6):1386-92. [PubMed: 15929775]

9. Otley A, et al. Assessing activity of pediatric Crohn's disease: which index to use? Gastroenterology. 1999; 116(3):527-31. [PubMed: 10029610]

10. Griffiths AM, et al. A review of activity indices and end points for clinical trials in children with Crohn's disease. Inflamm Bowel Dis. 2005; 11(2):185-96. [PubMed: 15677913]

11. Hyams JS, et al. Development and validation of a pediatric Crohn's disease activity index. J Pediatr Gastroenterol Nutr. 1991; 12(4):439-47. [PubMed: 1678008]

12. Turner D, et al. Appraisal of the pediatric ulcerative colitis activity index (PUCAI). Inflamm Bowel Dis. 2009

13. Sandler RS, Jordan MC, Kupper LL. Development of a Crohn's index for survey research. J Clin Epidemiol. 1988; 41(5):451-8. [PubMed: 3367175]

14. Lewis JD, et al. Use of the noninvasive components of the Mayo score to assess clinical response in ulcerative colitis. Inflamm Bowel Dis. 2008; 14(12):1660-6. [PubMed: 18623174]

15. Loonen HJ, et al. A critical assessment of items on the Pediatric Crohn's Disease Activity Index. J Pediatr Gastroenterol Nutr. 2003; 36(1):90-5. [PubMed: 12500002]

16. Shepanski MA, et al. Is an abbreviated Pediatric Crohn's Disease Activity Index better than the original? J Pediatr Gastroenterol Nutr. 2004; 39(1):68-72. [PubMed: 15187784]

17. Hyams J, et al. Evaluation of the pediatric crohn disease activity index: a prospective multicenter experience. J Pediatr Gastroenterol Nutr. 2005; 41(4):416-21. [PubMed: 16205508] 
Figure $1 \mathrm{~A}$ :

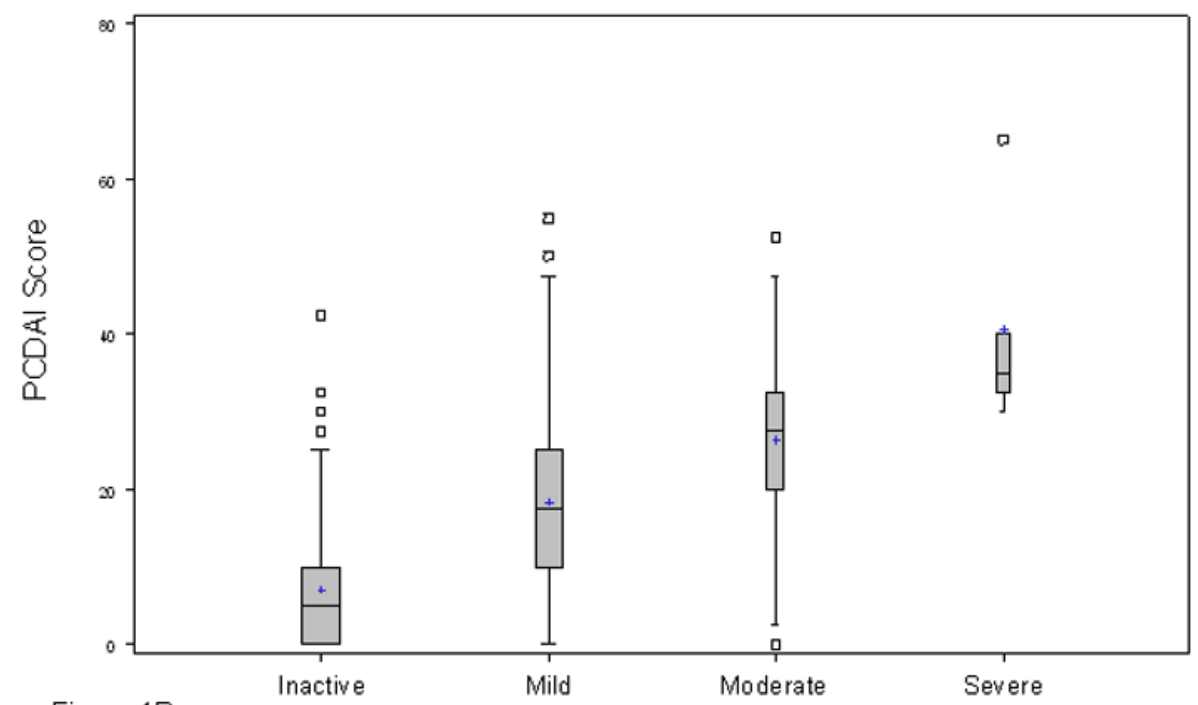

Figure 1B:

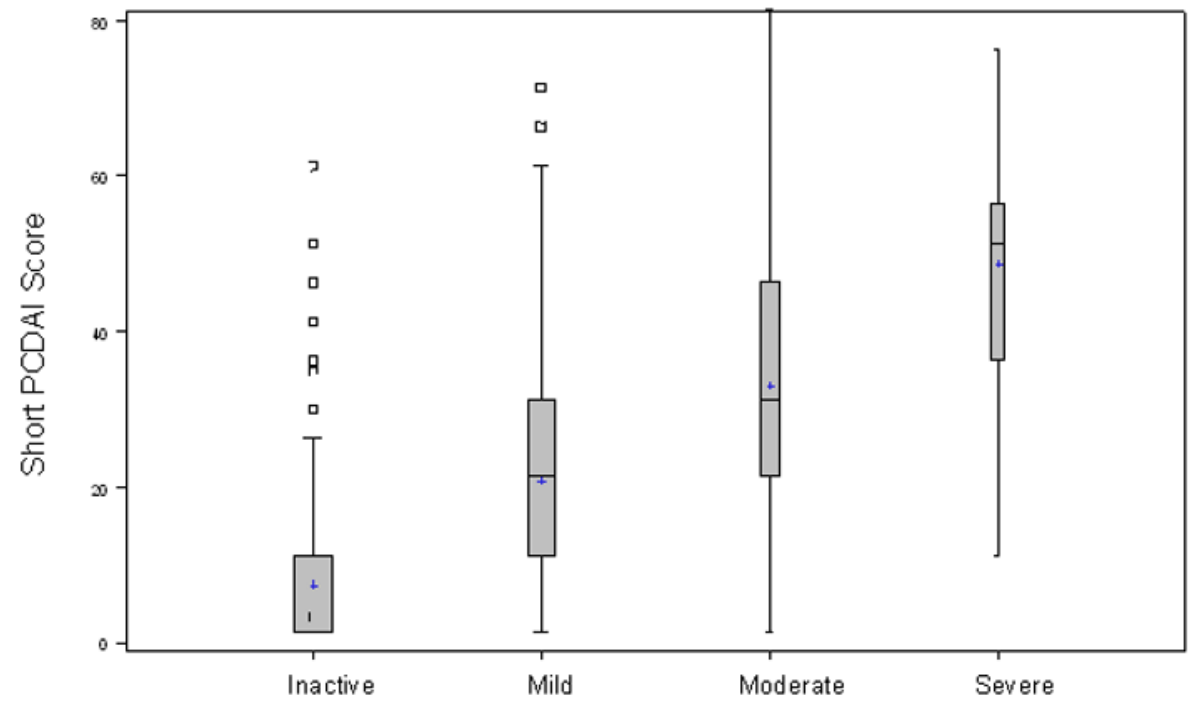

Figure 1.

"Distribution of Pediatric Crohn's Disease Activity Index (PCDAI) and Short PCDAI Scores According to Disease Severity as Measured by Physician Global Assessment" In these box and whisker plots, the + symbol denotes the mean activity index score for each category of disease severity, the vertical boxes depict the range of scores from the $25^{\text {th }}$ to $75^{\text {th }}$ percentile, and the horizontal hash mark in the middle of the vertical box represents median score. Figure 1A represents the PCDAI and Figure 1B represents the Short PCDAI. 
Table 1

Feasibility of PCDAI Components in Routine Clinical Care ( $\mathrm{N}=3,643$ visits $)$

\begin{tabular}{|l|l|}
\hline Component & \% visits completed \\
\hline Abdominal exam & 98 \\
\hline Abdominal pain & 96 \\
\hline Weight & 94 \\
\hline Extra-intestinal & 90 \\
\hline Stools & 88 \\
\hline Well-being & 84 \\
\hline ESR & 77 \\
\hline Albumin & 78 \\
\hline Hemoglobin & 68 \\
\hline Peri-rectal & 66 \\
\hline Height velocity & 63 \\
\hline Full PCDAI & 16.7 \\
\hline Abbreviated PCDAI & 44.1 \\
\hline
\end{tabular}


Table 2

Short PCDAI Components and Weights

\begin{tabular}{|c|c|}
\hline Component & Weight \\
\hline Abdominal pain & 20 points \\
\hline Well-being & 20 points \\
\hline Weight & 20 points \\
\hline Stool & 10 points \\
\hline Abdominal exam & 10 points \\
\hline Extraintestinal manifestations & 10 points \\
\hline Total & $\mathbf{9 0}$ points \\
\hline
\end{tabular}


Table 3

Correlation of Short PCDAI with other Indicators of Crohn's Disease Activity

\begin{tabular}{|c|c|c|c|}
\hline & PCDAI & Abbreviated PCDAI & Short PCDAI \\
\hline Log-transformed PCDAI & 0.83 & 0.68 & 0.66 \\
\hline Log-transformed Abbreviated PCDAI & 0.75 & 0.86 & 0.84 \\
\hline Log-transformed Short PCDAI & 0.72 & 0.83 & 0.86 \\
\hline PGA $^{*}$ & 0.61 & 0.64 & 0.60 \\
\hline Albumin $^{*}$ & 0.35 & 0.17 & 0.15 \\
\hline ESR $^{*}$ & 0.40 & 0.24 & 0.23 \\
\hline Hemoglobin $^{*}$ & 0.28 & 0.11 & 0.11 \\
\hline
\end{tabular}

Spearman correlation performed for ordinal variables 
Table 4

Area Under the Curve (AUC) for Short PCDAI versus other Clinical Indices

\begin{tabular}{|c|c|c|c|}
\hline Comparison & Short PCDAI AUC (95\% CI) & Abbreviated PCDAI AUC (95\% CI) & PCDAI AUC (95\% CI) \\
\hline Inactive vs Mild & $0.80(0.78,0.81)$ & $0.82(0.81,8.84)$ & $0.82(0.79,0.85)$ \\
\hline Mild vs Moderate-Severe & $0.72(0.69,0.75)$ & $0.72(0.68,0.84)$ & $0.72(0.66,0.78)$ \\
\hline
\end{tabular}


Table 5

Sensitivity and Specificity of Selected Short PCDAI Cut-points for Categories of Disease Severity by Physician Global Assessment

\begin{tabular}{|l|l|l|}
\hline Cut-point & Sensitivity & Specificity \\
\hline Inactive vs Mild & & \\
\hline$<5$ & 0.86 & 0.57 \\
\hline$<10$ & 0.83 & 0.62 \\
\hline$<15$ & 0.64 & 0.84 \\
\hline$<20$ & 0.57 & 0.88 \\
\hline Mod-Severe vs Mild & & \\
\hline$\geq 20$ & 0.81 & 0.43 \\
\hline$\geq 25$ & 0.70 & 0.63 \\
\hline$\geq 30$ & 0.64 & 0.73 \\
\hline$\geq 35$ & 0.52 & 0.84 \\
\hline
\end{tabular}

